\title{
Effect of paracentesis on the survival of patients with terminal cancer and ascites: A propensity score-weighted analysis of the East-Asian collaborative cross-cultural Study to Elucidate the Dying process
}

Ken Masuda ( $\square$ kenmasud@ncc.go.jp)

National Cancer Center Hospital: Kokuritsu Gan Kenkyu Center Chuo Byoin https://orcid.org/0000-0002-7736-3737

Hiroto Ishiki

National Cancer Center Hospital: Kokuritsu Gan Kenkyu Center Chuo Byoin https://orcid.org/0000-0002-0800-2161

Naosuke Yokomichi

Seirei Mikatahara Hospital: Seirei Mikatahara Byoin

Takuhiro Yamaguchi

Tohoku University Graduate School of Medicine

Tetsuya Ito

Japanese Red Cross Medical Center: Nihon Sekijujisha Iryo Center

Hana Takatsu

Konan Medical Center

Koji Amano

National Cancer Center

Shuji Hiramoto

Mitsubishi Kyoto Hospital

Toshihiro Yamauchi

Seirei Mikatahara Hospital: Seirei Mikatahara Byoin

Takashi Kawaguchi

Tokyo University of Pharmacy and Life Science: Tokyo Yakka Daigaku

Masanori Mori

Seirei Mikatahara Hospital: Seirei Mikatahara Byoin

Yosuke Matsuda

St. Lukes International Hospital

Takashi Yamaguchi

Konan Medical Center

Research Article

Keywords: ascites, terminal cancer, palliative care, end of life

Posted Date: February 4th, 2022

DOI: https://doi.org/10.21203/rs.3.rs-1208961/v1

License: (c) (i) This work is licensed under a Creative Commons Attribution 4.0 International License. Read Full License 


\section{Abstract}

Purpose: Paracentesis is among the most widely utilised treatments for Malignant ascites (MA). However, paracentesis in patients with MA has the potential to be associated with life-shortening effects. Thus, this study aimed to investigate whether paracentesis affected the duration of survival in such patients.

Methods: We performed a post-hoc analysis of a prospective multicenter observational study investigating the dying process and end-of-life care in patients with terminal cancer, admitted to 23 palliative care units in Japan. Survival duration was compared between patients who did (paracentesis group) and did not undergo paracentesis (non-paracentesis group). We used inverse probability of treatment weighting (IPTW) to control for baseline covariates between groups.

Results: Among the 1,896 initially enrolled patients, 568 with ascites were included in the study cohort. Eighty-five (15.0\%) patients underwent paracentesis. The primary tumor site was the pancreas $(51.9 \%, n=295)$, followed by the gastrointestinal tract $(22.7 \%, n=129)$. Non-adjusted median durations of survival were 22 days (95\% confidence interval [Cl]: 16-25) and 12 days (95\% $\mathrm{Cl}$ : 11-13) in the paracentesis and nonparacentesis groups, respectively (hazard ratio [HR]: $0.69,95 \% \mathrm{Cl}: 0.54-0.88 ; \mathrm{p}=0.003$ ). The IPTW-adjusted median survival durations were 22 (95\% Cl: $16-25)$ and 16 days (95\% Cl: $12-22)$ in the paracentesis and non-paracentesis groups, respectively (HR: 0.89, 95\% Cl: $0.64-1.24$; $\mathrm{p}=0.492)$. No serious adverse events occurred in the paracentesis group.

Conclusions: Paracentesis does not negatively affect the survival of patients with cancer and MA and can be a standard treatment in palliative care settings.

\section{Introduction}

Ascites is characterized by abnormal fluid accumulation in the peritoneal cavity. ${ }^{1}$ Malignant ascites (MA), which is mainly caused by peritoneal carcinomatosis, accounts for approximately $10 \%$ of all ascites cases. ${ }^{2,3}$ Intra-abdominal cancers, including pancreatic cancer, gastric cancer, uterine cancer, ovarian cancer, and malignant lymphoma, are common causes of MA and account for 30-54\% of all cases. ${ }^{4-7}$ Patients are typically in the palliative phase of care when they develop ascites. ${ }^{8}$ Thus, the optimal intervention should be both effective and have a minimal negative impact on quality of life. ${ }^{9}$

Patients with MA often experience distressing symptoms such as abdominal pain, bloating, loss of appetite, weight gain, impaired movement, fatigue, shortness of breath, and dyspnea. ${ }^{10-12}$ Moreover, MA is associated with progressive deterioration in quality of life and poor prognosis. $8,13,14$ Researchers have investigated several treatment options, including diuretics, paracentesis, tunneled catheters, intraperitoneal ports, peritoneovenous shunts, intraperitoneal catumaxomab, and hyperthermic intraperitoneal chemotherapy, for alleviating these symptoms. ${ }^{12}$ However, no standardized treatment for MA has been established because the evidence supporting the efficacy and safety of each approach is weak. 1,12

Given that it temporarily relieves symptoms in most patients, paracentesis is the most common therapeutic option for chemotherapy-resistant cases and is weakly recommended in the current guidelines. 1,9,15,16 Palliative paracentesis is frequently performed for patients with cancer in clinical practice; however, there is no high-quality evidence regarding its efficacy and feasibility in palliative care settings. In palliative care units (PCUs), the comfort of patients during their last days is more important than their life expectancy. However, in interventions that may have lifeshortening effects, survival is a common focus of ethical debate, and families, physicians, and nurses may have concerns and feelings of guilt about whether they might be hastening a patient's death. ${ }^{17,18,19}$ Therefore, determining whether paracentesis shortens the survival of patients with MA is critical for establishing the optimal symptom control strategy in these patients.

This study aimed to investigate the association between paracentesis and the survival of patients with MA using data from a large-scale, multicenter, prospective, observational study. We hypothesized that paracentesis would shorten the duration of survival in patients with terminal cancer.

\section{Methods}

\section{Study design and participants}

We performed a post-hoc analysis of a multicenter, prospective, observational cohort study known as the East-Asian collaborative cross-cultural Study to Elucidate the Dying process (EASED), which addresses the dying process and end-of-life care in patients with terminal cancer admitted to PCUs in Japan. ${ }^{20}$ The EASED enrolled and followed up participants from 23 palliative care institutions across Japan between January 1 , 2017 and June 30, 2018. Consecutive eligible patients were enrolled if they had been newly referred to the participating PCU during the study period. All interventions and observations were carried out within routine clinical practice. The inclusion criteria for the present study were as 
follows: (1) age $\geq 18$ years), (2) diagnosis of locally extensive or metastatic cancer (including hematological neoplasms), and (3) admission to the PCU. Patients who were scheduled for discharge within 1 week or those who did not want to be enrolled, were excluded.

Using the EASED data, we aimed to assess the impact of paracentesis on prognosis in patients with MA. The participants in this study were patients with ascites on admission to PCUs. Patients without symptoms caused by ascites on admission were not excluded, as they may have become symptomatic and undergone paracentesis during hospitalization.

The EASED study was performed in accordance with the ethical standards of the Helsinki Declaration and the ethical guidelines for epidemiological research presented by the Ministry of Health, Labour and Welfare in Japan. The study protocol was reviewed and approved by the local institutional review boards of all participating institutions. Written consent was waived in accordance with local regulations.

\section{Procedures}

In this study, we defined paracentesis as the removal of ascitic fluid from the abdominal cavity via a temporarily inserted needle or catheter to relieve abdominal pressure and alleviate ascites-related symptoms. ${ }^{1,10,21}$ This definition did not include diagnostic paracentesis. As part of routine clinical practice, paracentesis was performed when clinically indicated (i.e., mainly based on the patient's symptoms). The volume of paracentesis and whether additional artificial hydration or albumin infusion was performed was decided by the primary palliative care physician.

We collected data regarding patient's age, sex, primary tumor site, and the presence/absence of metastatic lesions at admission. Laboratory data (i.e., albumin, total bilirubin, creatinine, and C-reactive protein) had been recorded because many patients underwent routine blood tests at admission. Furthermore, we recorded the Karnofsky Performance Status (KPS), co-treatments (i.e., hydration volume and the use of diuretics, corticosteroids, opioids, and albumin infusion), and ascites features (i.e., gross appearance, volume of paracentesis, history of paracentesis before admission) on the day of the first paracentesis in the PCU. In addition, we used a numerical rating scale (NRS) to assess abdominal distension prior to paracentesis and on the following day. Adverse events that may have been caused by paracentesis were recorded in accordance with the Common Terminology Criteria for Adverse Events version 4.0. On the day of death, the date of death and number of paracentesis procedures performed during the PCU stay were recorded. All measurements were performed by patients' primary palliative care physicians during daily clinical practice using a structured data-collecting sheet designed for the study. Patients who were discharged were followed up for 6 months from baseline.

\section{Statistical analysis}

First, the characteristics of patients with MA were described, and their survival was compared with that of patients without MA using the logrank test.

Then, we constructed a propensity score (PS) model (the conditional probability of undergoing paracentesis) by selecting a set of confounders between treatment assignment (undergoing paracentesis) and outcome (survival from admission to death), based on a backdoor criterion using a directed acyclic diagram (DAG) that draws the causal network linking receiving paracentesis, survival, and other variables. ${ }^{22-25}$ The DAG included both measured variables at admission (i.e., patient characteristics [age, sex, KPS, primary tumor site, and liver metastasis], complications [malignant bowel obstruction], laboratory data [albumin, C-reactive protein, total bilirubin, and creatinine], presence of symptoms owing to ascites, and opioid consumption) and an unmeasured variable (i.e., ascites volume). Variables selected as confounders (see Figure, Supplemental Digital Content 1, which demonstrates these variables). Albumin and C-reactive protein were used as markers of cachexia, while total bilirubin and creatinine were used as markers of hepatic and renal failure, respectively.

Next, under the missing at random assumption, we performed multiple imputations by chain equations to impute missing values for KPS (0.1\%), albumin (12.7\%), total bilirubin (13.6\%), creatinine (11.4\%), and C-reactive protein (13.6\%). ${ }^{27}$ The variables included in the imputation models were the same as those in the PS model. In total, ten complete datasets were generated for subsequent analyses.

We compared baseline characteristics between patients who did not undergo paracentesis (non-paracentesis group) and those who did (paracentesis group). The balance in covariates was assessed using the standardized mean difference (SMD). SMD $>0.1$ was interpreted as a meaningful difference.

To account for selection and confounding biases, the observed differences in baseline covariates between the two groups were adjusted using the inverse probability of treatment weighting (IPTW) method. ${ }^{23,24}$ In this method, the PS for each patient was estimated using multivariate logistic regression of the PS model. Subsequently, the PS values from the ten imputed datasets were pooled according to Rubin's rule. ${ }^{27}$ Finally, scores in the non-paracentesis group were weighted by the average treatment effect for treated weight: [PS/(1-PS)]. This method produces a weighted pseudo-sample of patients in the reference group with the same distribution of measured covariates as in the exposed group. 
Survival was calculated from the day of admission to the day of death. Adjusted Kaplan-Meier curves were computed based on inverse probability weights, and a univariate inverse probability-weighted Cox proportional hazards model was used to estimate the IPTW-adjusted hazard ratio (HR) for patient survival in the paracentesis and non-paracentesis groups. Furthermore, subgroup analyses were performed to investigate the IPTW-adjusted HR of the paracentesis and non-paracentesis groups according to some of the baseline covariates, including age, sex, KPS, primary tumor site, liver dysfunction (defined as total bilirubin higher than $4.0 \mathrm{mg} / \mathrm{dL}$ at admission), and renal dysfunction (defined as creatinine higher than $1.5 \mathrm{mg} / \mathrm{dL}$ at admission).

Finally, two sensitivity analyses were conducted to assess the robustness of the results. First, 265 patients without symptoms of ascites at admission were excluded to determine whether results varied according to patient selection. Second, patients who were discharged alive were excluded to determine whether results were affected by the censored population.

All statistical analyses were performed using R version 3.5.3 (R Core Team 2019, Vienna, Austria). All p-values were two-sided, and p-values $<0.05$ were considered significant. Imputation of missing data was conducted using the "MICE" package, and survival analyses were performed using the "survival" package.

\section{Role of the funding source}

This work was supported by Grant-in-Aid from the Japanese Hospice Palliative Care Foundation and JSPS KAKENHI (Grant Number JP20K16567).

\section{Results}

\section{Patient characteristics}

A total of 1,971 patients were assessed for eligibility (Figure 1). After excluding 45 patients who were prescheduled for discharge within a week and three who personally or whose families declined participation, 1,926 patients with advanced cancer were enrolled. Among those patients, 30 were excluded because they were lost to follow-up, and 1,328 were excluded because they had no ascites. Thus, the population for analysis included 568 patients.

The patient characteristics after imputation are summarized in Table 1. The median age was 71 years, and $55.6 \%$ of patients were male. The most common primary tumor site was the gastrointestinal tract (51.9\%), followed by the pancreas (22.7\%) and ovaries (19.5\%). The median KPS was 40 . Symptoms of ascites were present in $53.3 \%$ of patients. The median Memorial Delirium Assessment Scale score was 0.0 .

The median duration of survival from admission among the 568 patients with ascites was 13 days (95\% confidential interval [Cl]: 12-14), which was significantly shorter than that among the 1,328 patients without ascites ( 21 days, 95\% Cl: 19-23; log-rank p<0.0001; (see Figure,

Supplemental Digital Content 2).

\section{Characteristics of paracentesis}

Among the 568 included patients, 85 underwent paracentesis during their PCU stay. The characteristics of the patients that underwent paracentesis are summarized in Table 2. Paracentesis was performed prior to enrolment in $62.4 \%$ of patients. The median time from admission to the day of paracentesis was 3 days (interquartile range [IQR]: 1-7 days). The median number of paracentesis procedures performed during the PCU stay was 2 (IQR: 1-3), and the median volume of the removed ascites was $2000 \mathrm{~mL}$ (IQR: $1700-3000 \mathrm{~mL}$ ). Serous, hemorrhagic, and chylous ascites were present in $80 \%, 14 \%$, and $7 \%$ of patients, respectively. More than half of the patients received no additional artificial hydration during or after paracentesis, and $5.9 \%$ of patients received albumin infusion during paracentesis. The concomitant medications used by patients on admission included diuretics (35.7\%), corticosteroids (21.4\%), and opioids (50.6\%). The median NRS values for abdominal distension before and on the day following paracentesis were 7.5 and 4.0, respectively. There were no adverse events with grades higher than 3. Mild ascitic leakage and hypotension developed in $8.1 \%$ and $5.3 \%$ of patients, respectively. Three patients (3.5\%, 95\% Cl: $0.7-10)$ died within 3 days after paracentesis.

\section{Balance between covariates of the non-paracentesis and paracentesis groups}

Comparisons of the baseline covariates between the two groups are presented in Table 1. Patients in the paracentesis group were significantly younger, had better performance status, experienced more frequent symptoms due to ascites, experienced less severe agitated delirium, had higher serum albumin levels, and had lower C-reactive protein and creatinine levels than those in the non-paracentesis group. There were no significant differences in age, sex, or primary tumor site between the two groups. After PS weighting, the standardized difference for each covariate was $<0.1$, indicating that the weighted population in the two groups was comparable.

\section{Patient survival according to paracentesis}


Figure 2 shows the Kaplan-Meier curves for survival after admission to the PCU according to paracentesis in the non-adjusted and IPTWadjusted cohorts. The non-adjusted median durations of survival were 22 days (95\% Cl: $16-25)$ and 12 days (95\% Cl: $11-13)$ in the paracentesis and non-paracentesis groups, respectively (HR 0.69, 95\% Cl: 0.54-0.88; $\mathrm{p}=0.0027)$. The IPTW-adjusted median durations of survival were 22 days (95\% Cl: $16-25)$ and 16 days (95\% Cl: $12-22)$ in the paracentesis and non-paracentesis groups, respectively (HR 0.89 , 95\% Cl: 0.64-1.24; $p=0.49)$.

\section{Subgroup analysis}

To compare survival between the paracentesis and non-paracentesis groups, we performed a weighted subgroup analysis according to baseline covariates including age, sex, KPS, primary tumor site, liver dysfunction, and renal dysfunction. There was no significant heterogeneity across all subgroups. Paracentesis was not associated with a significant survival risk in any of the subgroups (Figure 3).

\section{Sensitivity analyses}

After excluding the 265 patients whose ascites was asymptomatic at admission, survival did not significantly differ between the groups (HR: $0.86,95 \%$ Cl: 0.61-1.21; $p=0.38$ ) (see Figure, Supplemental Digital Content 3A). Furthermore, after excluding the 52 patients who were discharged alive from the PCU, survival did not significantly differ between the groups (HR: 0.75, 95\% Cl: 0.54-1.05; p=0.091) (see Figure, Supplemental Digital Content 3B).

\section{Discussion}

This large-scale, multicenter, prospective, observational study revealed that survival among patients with terminal cancer and MA did not significantly differ based on whether paracentesis was performed. Most importantly, after adjustment for potential confounders using the IPTW method, our results indicated that paracentesis does not negatively affect the survival of patients with advanced cancer and MA. There was no significant heterogeneity across all subgroups of age, sex, primary tumor site, KPS, liver dysfunction, and renal dysfunction. This indicates that even patients with poor performance status or impaired organ function might be able to safely undergo paracentesis. Furthermore, two sensitivity analyses (i.e., analysis of patients with symptomatic ascites only and analysis excluding patients discharged alive) confirmed the result. Paracentesis was generally well tolerated, with no associated deaths, and it also reduced the symptoms of ascites. A median of $2000 \mathrm{~mL}$ of ascites could be safely removed from patients with advanced cancer in the PCU. The findings of this study support the recommendations for paracentesis in the guidelines for the management of $\mathrm{MA}^{1,19}$ and can relieve the concerns of patients, families, and physicians that paracentesis may shorten survival.

Notably, the survival rate was significantly poorer in patients with MA than in those without MA, consistent with the findings of previous studies. ${ }^{5,14}$ Ayantunde et al. evaluated the pattern of cancers causing MA and factors affecting survival by retrospectively reviewing the data of 209 patients with various cancers. ${ }^{14}$ In their study, paracentesis was performed in 112 patients, and diuretics and chemotherapy were administered to 70 and 103 patients, respectively. The median survival following the diagnosis of ascites was 5.7 months (95\% Cl: 3.54-7.93). Patients with ovarian cancer had a survival of nearly 2 years, while patients with other cancers, such as gastrointestinal cancers, had a survival of less than 3 months. In 1982, Appelqvist et al. reviewed a series of 100 consecutive cases of malignancy treated for ascites with abdominal paracentesis. ${ }^{5}$ They reported that the median duration of survival of patients with carcinoma of the mammary gland, ovary, and large intestine were 47, 121, and 54 days after the first abdominal paracentesis, respectively. Although the populations of these two studies differed from our own, the prognosis for patients with MA was also relatively poor despite treatment with abdominal paracentesis. However, the above-mentioned studies did not examine the impact of puncture on prognosis, and high-quality clinical studies, such as randomized clinical trials (RCTs) have not been performed to date.

RCTs are the gold standard for comparing and demonstrating the impact of ascites paracentesis on prognosis. However, since the symptoms associated with ascites retention are quickly resolved by paracentesis, it is difficult to perform RCTs in end-of-life patients due to ethical issues and high attrition rates. ${ }^{28,29}$ Our study, instead compared whether or not to perform paracentesis by carefully eliminating several confounders. Moreover, the data were prospectively collected, and our large cohort was derived from 22 facilities, suggesting that our results are generalizable. In addition, we removed confounding factors using the PS-weighting method and confirmed that there were no large differences in missing values using multiple correction methods. As the effects of anti-cancer treatment (e.g., chemotherapy) may affect the study of ascites, the study was conducted among PCU patients receiving no concomitant anti-cancer treatment, thus confirming the effects of paracentesis at the end of life in patients with MA. We also confirmed that ascites puncture can be safely performed in an extremely vulnerable population with a median survival of 22 days.

This study had several limitations. First, because this was not an interventional study, the patient population is heterogeneous and causal factors of MA were not considered. There may have been unmeasured confounders (e.g., cardiopulmonary function, symptom severity, patient's request for a puncture, and refractoriness to medical treatment). We believe, however, that this is acceptable because almost all admitted patients were enrolled in the study, and the obtained data reflect real-world practice. Thus, we adjusted for major-specific confounders using 
DAG. Second, the patient selection process may have affected our results because we included patients with asymptomatic ascites at admission. However, given that we adjusted for the presence of ascites symptoms in the main analysis, we do not believe that this significantly influenced the results. Furthermore, the results were robust when sensitivity analyses were performed while excluding patients with no symptoms of ascites at admission. Third, we did not consider the patients' history of paracentesis before admission. Nevertheless, we believe that this is acceptable because our main focus was whether paracentesis shortens the survival of patients with terminal cancer. Lastly, our findings cannot be generalized to patients who have not been admitted to a PCU or those who have undergone paracentesis while in a better general condition. The effect of draining larger amounts than those drained in the present study remains unknown, and it is unclear from our results whether it is better to drain a large amount of fluid in a single instance or smaller amounts over multiple punctures. Our results may have differed if we had collected data from patients in general wards. Further studies are warranted to clarify the association between paracentesis and survival of these populations. Despite these limitations, we believe that our finding that paracentesis does not negatively affect survival is highly meaningful, having important implications for clinical practice.

\section{Conclusions}

The present findings demonstrate that paracentesis is unlikely to shorten the survival of patients with terminal cancer and MA. Hence, health care professionals need not hesitate in deciding whether to conduct paracentesis in terminally ill patients with symptomatic MA, as it is not associated with life-shortening effects, but is rather associated with alleviating MA symptoms.

\section{Declarations}

\section{Funding}

This work was supported by Grant-in-Aid from the Japanese Hospice Palliative Care Foundation and JSPS KAKENHI (Grant Number JP20K16567).

\section{Conflicts of interest/Competing interests}

Dr. Ishiki reports personal fees from Mundipharma, Morinagaclinico, Merckserono, and GuerbetJapan and non-financial support from Shionogi outside of the submitted work; Dr. Masuda reports personal fees from Chugai and Astrazeneca outside of the submitted work. All other authors state that they have no conflicts of interest.

\section{Availability of data and material}

The datasets generated during the current study are not publicly available due to ethical restrictions but are available from the corresponding author upon reasonable request.

\section{Code availability}

Not applicable

\section{Authors' contributions}

$\mathrm{KM}, \mathrm{HI}, \mathrm{TI}, \mathrm{NY}, \mathrm{TK}$, and MM made substantial contributions to the conception and design of the study, acquisition of data, and data analysis. KM and $\mathrm{HI}$ drafted the manuscript and approved the submitted version. $\mathrm{HI}$ made substantial contributions to the study design and revision of the manuscript. NY and TY accessed and verified the underlying study data and contributed to data analysis and interpretation. TI, HT, KA, SH, TY, YM, and TY contributed to the conception and study design, data collection and assembly, data interpretation, writing of the manuscript, and critical revision of the manuscript for important intellectual content. All authors have read and approved the final manuscript.

\section{Ethics approval}

The EASED study was performed in accordance with the ethical standards of the Helsinki Declaration and the ethical guidelines for epidemiological research presented by the Ministry of Health, Labour and Welfare in Japan. The study protocol was reviewed and approved by the local institutional review boards of all participating institutions.

\section{Consent to participate}

Japanese law does not require individual informed consent from participants in a non-invasive observational trial. Therefore, we used an opt-out method rather than acquiring written or oral informed consent.

\section{Consent for publication}


Not applicable

\section{Acknowledgements}

We highly appreciate the participation of the patients and their families, and the cooperation of members of the East-Asian collaborative crosscultural Study to Elucidate the Dying process (EASED) study group.

\section{References}

1. Becker G, Galandi D, Blum HE. Malignant ascites: systematic review and guideline for treatment. Eur J Cancer. 2006;42:589-597.

2. Runyon BA. Care of patients with ascites. N Engl J Med. 1994;330:337-342.

3. Runyon BA. Malignancy-related ascites and ascitic fluid "humoral tests of malignancy". J Clin Gastroenterol. 1994;18:94-98.

4. Wilailak S, Linasmita V, Srivannaboon S. Malignant ascites in female patients: a seven-year review. J Med Assoc Thai. 1999;82:15-19.

5. Appelqvist P, Silvo J, Salmela L, et al. On the treatment and prognosis of malignant ascites: is the survival time determined when the abdominal paracentesis is needed? J Surg Oncol. 1982;20: 238-242.

6. Ringenberg QS, Doll DC, Loy TS, et al. Malignant ascites of unknown origin. Cancer. 1989; 64:753-755.

7. Mackey JR, Venner PM. Malignant ascites: demographics, therapeutic efficacy and predictors of survival. Can J Oncol. 1996;6:474-480.

8. Garrison RN, Kaelin LD, Galloway RH, et al. Malignant ascites. Clinical and experimental observations. Ann Surg. 1986;203:644-651.

9. Lee CW, Bociek G, Faught W. A survey of practice in management of malignant ascites. J Pain Symptom Manag. 1998;16:96-101.

10. Chung M, Kozuch P. Treatment of malignant ascites. Curr Treat Options Oncol. 2008;9:215-233.

11. Sangisetty SL, Miner TJ. Malignant ascites: A review of prognostic factors, pathophysiology and therapeutic measures. World $J$ Gastrointest Surg. 2012;4:87-95.

12. Hodge C, Badgwell BD. Palliation of malignant ascites. J Surg Oncol. 2019;120:67-73.

13. Saif MW, Siddiqui IA, Sohail MA. Management of ascites due to gastrointestinal malignancy. Ann Saudi Med. 2009;29:369-377.

14. Ayantunde AA, Parsons SL. Pattern and prognostic factors in patients with malignant ascites: a retrospective study. Ann Oncol. 2007;18:945-949.

15. Jehn CF, Küpferling S, Oskay-Özcelik G, et al. A survey of treatment approaches of malignant ascites in Germany and Austria. Support Care Cancer. 2015;23:2073-2078.

16. Hisanaga T, Shinjo T, Imai K, et al. Clinical Guidelines for Management of Gastrointestinal Symptoms in Cancer Patients: The Japanese Society of Palliative Medicine Recommendations. J Palliat Med. 2019;22:986-997.

17. Maeda I, Morita T, Yamaguchi T, et al. Effect of continuous deep sedation on survival in patients with advanced cancer (J-Proval): a propensity score-weighted analysis of a prospective cohort study. Lancet Oncol. 2016;17:115-122.

18. Materstvedt LJ, Bosshard G. Deep and continuous palliative sedation (terminal sedation): clinical-ethical and philosophical aspects. Lancet Oncol. 2009;10:622-627.

19. Papavasiliou EE, Payne S, Brearley S, et al. Current debates on end-of-life sedation: an international expert elicitation study. Support Care Cancer. 2014;22:2141-2149.

20. Hiratsuka Y, Yamaguchi T, Maeda I, et al. The Functional Palliative Prognostic Index: a scoring system for functional prognostication of patients with advanced cancer. Support Care Cancer. 2020;28:6067-6074.

21. Wimberger $\mathrm{P}$, Gilet $\mathrm{H}$, Gonschior $\mathrm{AK}$, et al. Deterioration in quality of life (QoL) in patients with malignant ascites: results from a phase II/III study comparing paracentesis plus catumaxomab with paracentesis alone. Ann Oncol. 2012;23:1979-1985.

22. Greenland S, Pearl J, Robins JM. Causal diagrams for epidemiologic research. Epidemiology. 1999;10:37-48.

23. Austin PC, Stuart EA. Moving towards best practice when using inverse probability of treatment weighting (IPTW) using the propensity score to estimate causal treatment effects in observational studies. Stat Med. 2015;34:3661-3679.

24. Desai RJ, Franklin JM. Alternative approaches for confounding adjustment in observational studies using weighting based on the propensity score: a primer for practitioners. BMJ. 2019;367:I5657.

25. Hernán MA RJ. Causal Inference: What If. CRC: Boca Raton: Chapman \& Hall; 2020.

26. White IR, Royston P, Wood AM. Multiple imputation using chained equations: Issues and guidance for practice. Stat Med. 2011;30:377-399.

27. Rubin DB. Multiple imputation for nonresponse in surveys. John Wiley \& Sons; 2004.

28. Preston NJ, Fayers P, Walters SJ, et al. Recommendations for managing missing data, attrition and response shift in palliative and end-oflife care research: part of the MORECare research method guidance on statistical issues. Palliat Med. 2013;27:899-907. 
29. Hui D, Glitza I, Chisholm G, et al. Attrition rates, reasons, and predictive factors in supportive care and palliative oncology clinical trials. Cancer. 2013;119:1098-1105.

\section{Tables}

Table 1. Characteristics of patients who received paracentesis versus those who did not in unweighted and weighted cohorts 
Variable

Unweighted Cohort

$\begin{array}{lll}\text { Total } & \begin{array}{l}\text { Non- } \\ \text { paracentesis }\end{array} & \text { Paracentesis } \\ (\mathrm{N}= & (\mathrm{N}=483) & (\mathrm{N}=85)\end{array}$

$(\mathrm{N}=$
$568)$$\quad(\mathrm{N}=483) \quad(\mathrm{N}=85)$
Propensity Score-Weighted Cohort ${ }^{a}$

Non-paracentesis Paracentesis

SMD $^{b}$

$(\mathrm{N}=83.4)$

$(\mathrm{N}=85)$

SMD $^{b}$

Patient

characteristics

Age (median

[IQR])

$71.0 \quad 71.0 \quad 69.0[61.0,79.0]$

[63.0, [64.0,

$-0.22$

$67.0[59.0$,

$69.0[61.0,79.0]$

0.014

$\begin{array}{lll}\text { Sex, female (\%) } & 316 & 267 \\ & (55.6) & (55.3)\end{array}$

49 (57.6)

$0.024 \quad 49.5(59.3)$

49 (57.6)

$-0.017$

Primary tumor

site (\%)

Gastrointestinal

tract $\quad$ (51.9)

Pancreas

$(51.9)(52.4)$

$42(49.4)$

$-0.030 \quad 41.0(49.2)$

$42(49.4)$

$-0.021$

$129 \quad 113$

16 (18.8)

$-0.046 \quad 18.6(22.3) \quad 16(18.8)$

0.0035

$\begin{array}{lll}\text { Ovary } & 111 & 91 \\ & (19.5) & (18.8)\end{array}$

(22.7) (23.4)

Others

33.26

20 (23.5)

$0.047 \quad 16.9(20.2) \quad 20(23.5)$

0.033

$\begin{array}{llll} & (5.8) & (5.4) & \\ & & & \\ \text { KPS (median } & 40.0 & 40.0 & 40.0[40.0,50.0] \\ {[\text { IQR]) }} & {[30.0,} & {[30.0,} & \\ & 50.0] & 50.0] & \end{array}$

$0.029 \quad 6.9(8.3) \quad 7(8.2)$

$-0.0002$

KPS (median

$\begin{array}{ll}{[30.0,} & {[30.0,} \\ 50.0] & 50.0]\end{array}$

$0.72 \quad 50.0[40.0$

$40.0[40.0,50.0]$

0.037

Symptoms

\begin{tabular}{|c|c|c|c|c|c|c|c|}
\hline $\begin{array}{l}\text { Symptoms due } \\
\text { to ascites, } \\
\text { present (\%) }\end{array}$ & $\begin{array}{l}303 \\
(53.3)\end{array}$ & $\begin{array}{l}226 \\
(46.8)\end{array}$ & 77 (90.6) & 0.44 & $75.5(90.6)$ & 77 (90.6) & 0.0002 \\
\hline $\begin{array}{l}\text { Severity of } \\
\text { agitated } \\
\text { delirium } \\
\text { (median [IQR]) }^{c}\end{array}$ & $\begin{array}{l}0.0 \\
(0.0)\end{array}$ & $\begin{array}{l}0.0 \\
{[0.0} \\
0.0]\end{array}$ & $0.0[0.0,0.0]$ & -0.31 & $0.0[0.0,0.0]$ & $0.0[0.0,0.0]$ & -0.0086 \\
\hline \multicolumn{8}{|l|}{$\begin{array}{l}\text { Laboratory } \\
\text { Variables }\end{array}$} \\
\hline $\begin{array}{l}\text { Albumin }-\mathrm{g} / \mathrm{dL} \\
\text { (median [IQR]) }\end{array}$ & $\begin{array}{l}2.20 \\
{[1.80} \\
2.60]\end{array}$ & $\begin{array}{l}2.20 \\
{[1.80} \\
2.60]\end{array}$ & $2.30[1.80,2.60]$ & 0.17 & $\begin{array}{l}2.20[1.90, \\
2.60]\end{array}$ & $2.30[1.80,2.60]$ & 0.068 \\
\hline $\begin{array}{l}\text { C-reactive } \\
\text { protein -mg/dL } \\
\text { (median [IQR]) }\end{array}$ & $\begin{array}{l}0.90 \\
{[0.50} \\
2.50]\end{array}$ & $\begin{array}{l}0.90 \\
{[0.50} \\
2.60]\end{array}$ & $0.80[0.60,2.00]$ & -0.32 & $\begin{array}{l}0.80[0.50, \\
2.60]\end{array}$ & $0.80[0.60,2.00]$ & -0.050 \\
\hline $\begin{array}{l}\text { Total bilirubin - } \\
\text { mg/dL (median } \\
{[\mathrm{IQR}] \text { ) }}\end{array}$ & $\begin{array}{l}0.86 \\
{[0.60} \\
1.29]\end{array}$ & $\begin{array}{l}0.84 \\
{[0.58} \\
1.29]\end{array}$ & $0.97[0.68,1.30]$ & 0.059 & $\begin{array}{l}0.76[0.59, \\
1.16]\end{array}$ & $0.97[0.68,1.30]$ & 0.035 \\
\hline $\begin{array}{l}\text { Creatinine - } \\
\text { mg/dL (median } \\
\text { [IQR]) }\end{array}$ & $\begin{array}{l}5.29 \\
{[2.45} \\
11.76]\end{array}$ & $\begin{array}{l}5.64 \\
{[2.48} \\
11.87]\end{array}$ & $4.04[2.21,9.84]$ & -0.19 & $\begin{array}{l}4.50[2.26, \\
8.70]\end{array}$ & $4.04[2.21,9.84]$ & 0.040 \\
\hline
\end{tabular}

$\begin{array}{lll}\mathrm{mg} / \mathrm{dL} & (\text { median } & {[2.45} \\ {[\mathrm{IQR}]} & 11.76] & 11.87\end{array}$

Abbreviation: SMD: standardized mean difference; IQR: interquartile range. KPS: Karnofsky Performance Status

a Weighted using inverse probability of treatment weighting, based on propensity scores. Patients in non-paracentesis group were weighted by the average treatment effect for treated (ATT) weight.

b The mean value of SMD across 10 imputed datasets. An absolute SMD greater than 0.1 is interpreted as a meaningful difference.

${ }^{c}$ Assessed using Memorial Delirium Assessment Scale item 9, rated from 0 (normal) to 3 (severe).

Table 2. Characteristics of the first paracentesis during PCU stay 


\begin{tabular}{|c|c|}
\hline Variable & $N=85$ \\
\hline Received paracentesis before enrollment (\%) & $53(62.4)$ \\
\hline Time from enrollment to paracentesis (days, median [IQR]) & $3.0[1.0,7.0]$ \\
\hline Number of paracentesis during PCU stay (median [IQR]) & $2.0[1.0,3.0]$ \\
\hline Volume of drained ascitic fluid (ml, median [IQR]) & $2000[1700,3000]$ \\
\hline \multicolumn{2}{|l|}{ Gross appearance of ascites } \\
\hline Serous (\%) & $68(80.0)$ \\
\hline Hemorrhagic (\%) & $12(14.1)$ \\
\hline Chylous (\%) & $6(7.1)$ \\
\hline \multicolumn{2}{|l|}{ Co-treatment } \\
\hline Hydration volume (median [IQR]) & $0.0[0.0,275.0]$ \\
\hline Diuretics use (\%) & $30(35.7)$ \\
\hline Corticosteroid use (\%) & $18(21.4)$ \\
\hline Opioid use (\%) & $43(50.6)$ \\
\hline Albumin infusion (\%) & $5(5.9)$ \\
\hline \multicolumn{2}{|l|}{ Efficacy } \\
\hline \multicolumn{2}{|l|}{ Abdominal distension NRS (median [IQR]) ${ }^{a}$} \\
\hline pre-paracentesis & $7.5[6.0,8.0]$ \\
\hline post-paracentesis & $4.0[2.0,5.0]$ \\
\hline \multicolumn{2}{|l|}{ Adverse event ${ }^{b}$} \\
\hline Ascites leakage (\%) & $6(8.1)$ \\
\hline Hypotension (\%) & $4(5.3)$ \\
\hline Bleeding (\%) & $0(0.0)$ \\
\hline Infection (\%) & $0(0.0)$ \\
\hline Perforation (\%) & $0(0.0)$ \\
\hline $\begin{array}{l}\text { Abbreviation: PCU: palliative care unit; IQR: interquartile rang } \\
{ }^{a} n=48 \\
{ }^{b} n=74 \text {. No grade }>=3 \text { event was observed. }\end{array}$ & numerical rating sca \\
\hline
\end{tabular}

\section{Figures}




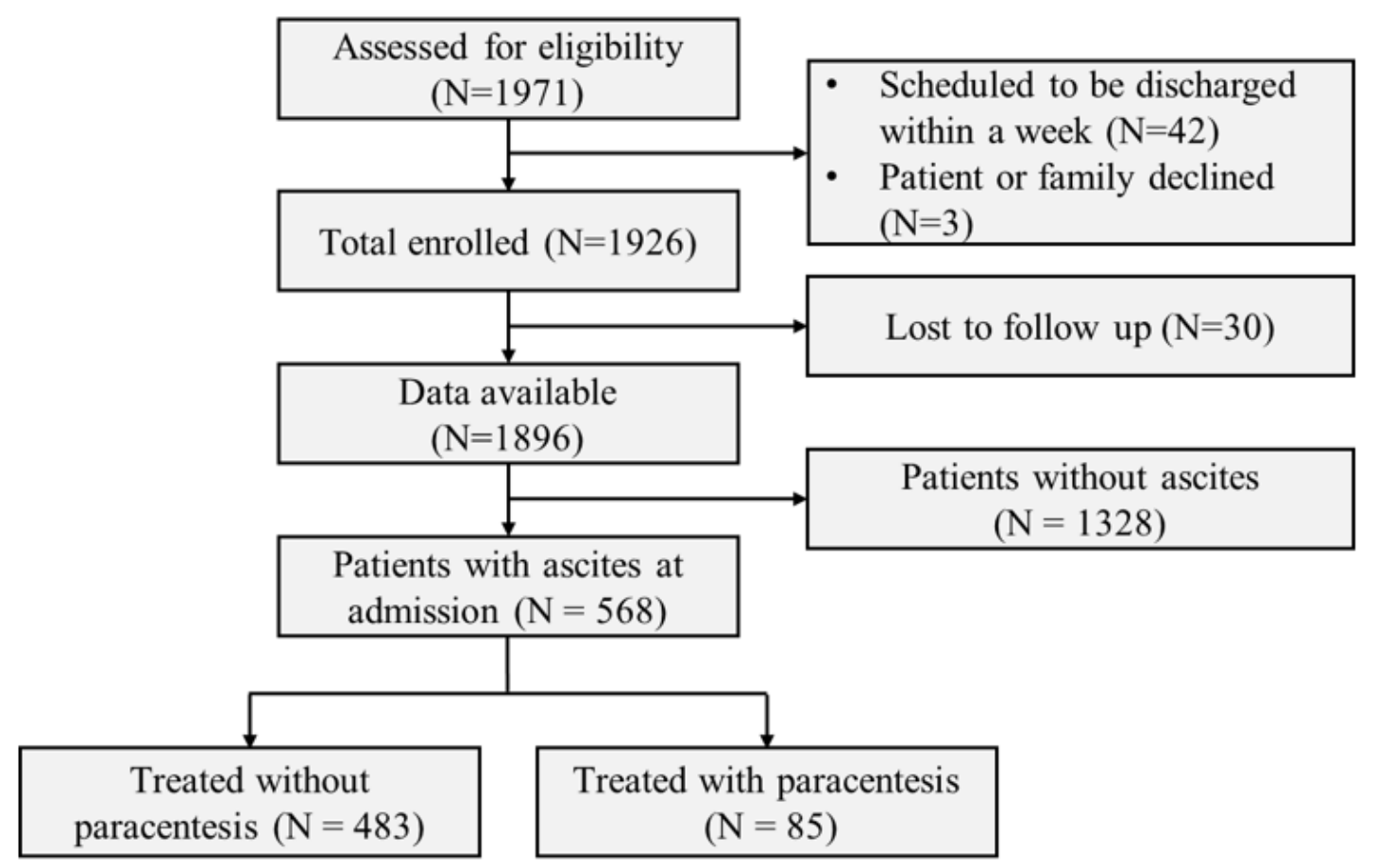

Figure 1

Patient selection flow chart per the Strengthening the Reporting of Observational Studies in Epidemiology (STROBE) statement.

A) Unweighted cohort

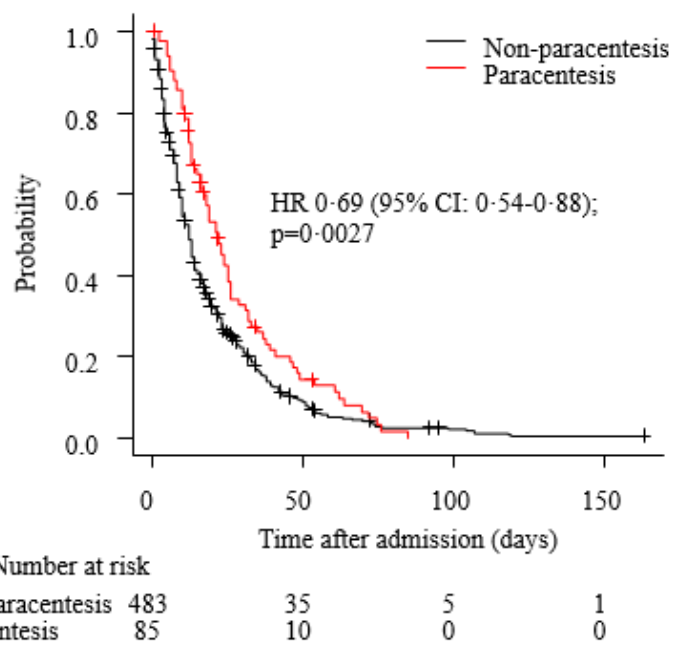

B) Propensity score-weighted cohort

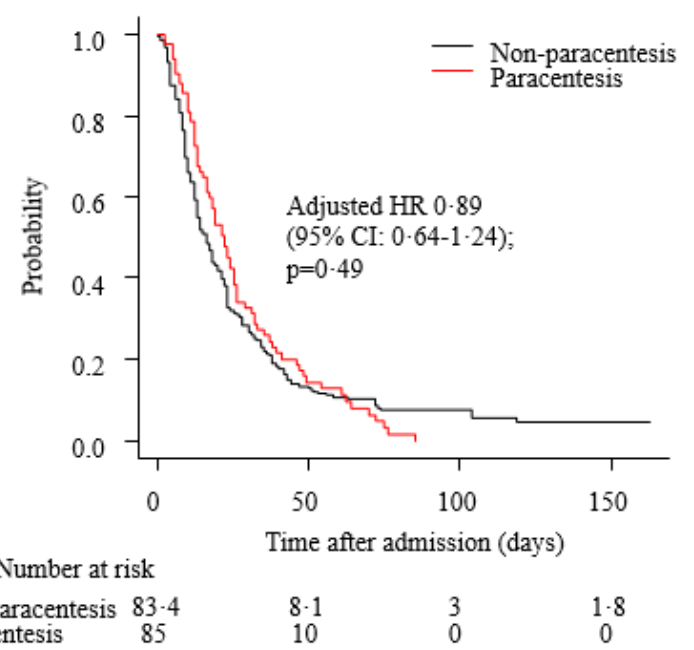

Figure 2

Comparison of survival between the non-paracentesis and paracentesis groups. (A) Unweighted cohort; (B) Propensity score-weighted cohort. $\mathrm{HR}$, hazard ratio; $\mathrm{Cl}$, confidence interval 


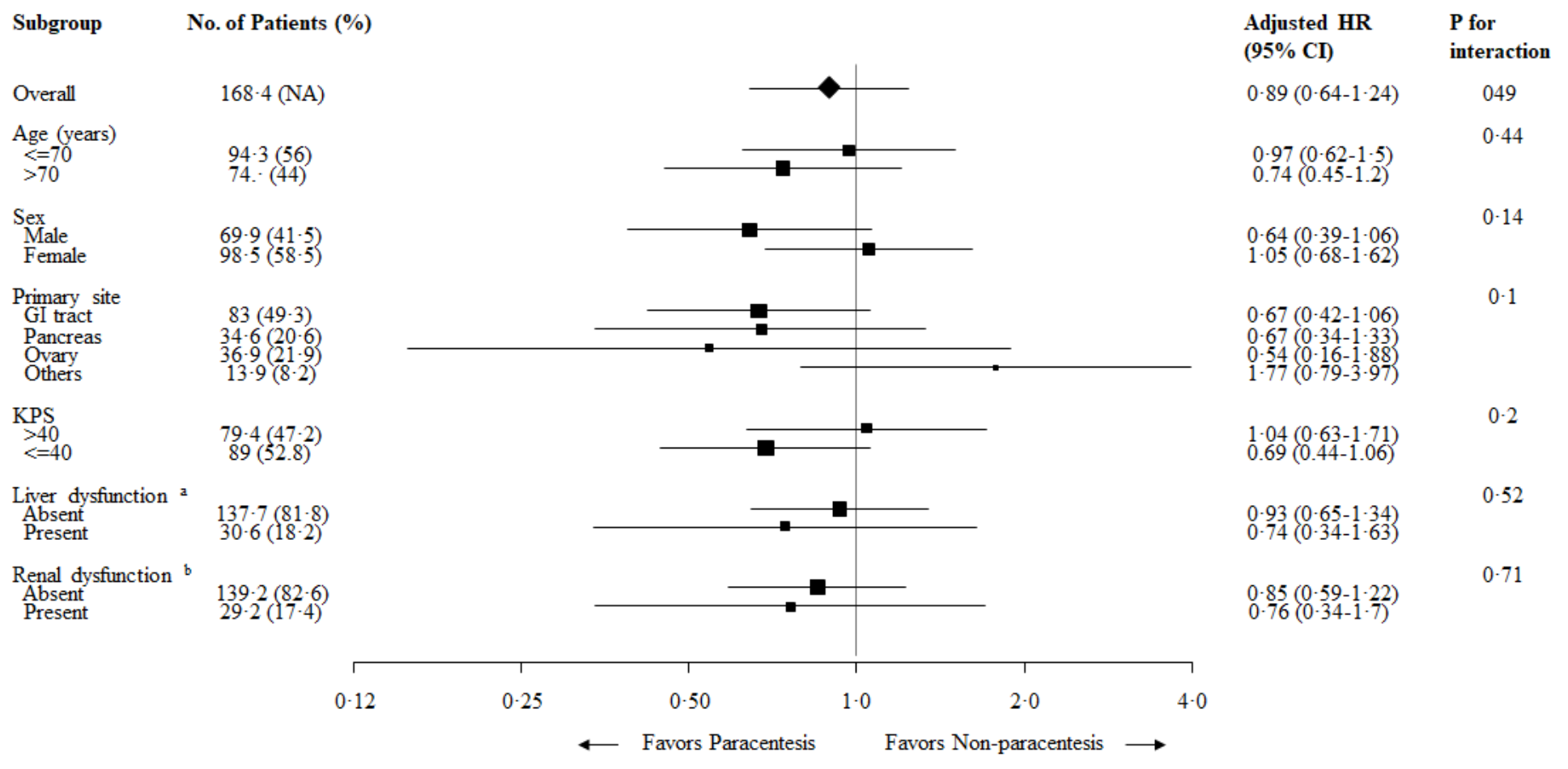

\section{Figure 3}

Weighted subgroup analysis of survival in patients with and without paracentesis. IPTW-adjusted hazard ratios were calculated using a Cox proportional hazards model after stratification into subgroups.

${ }^{a}$ Defined as a total bilirubin level of more than $4.0 \mathrm{mg} / \mathrm{dL}$.

${ }^{b}$ Defined as creatinine level of more than $1.5 \mathrm{mg} / \mathrm{dL}$.

Abbreviations: HR, hazard ratio; Cl, confidence interval; GI, gastrointestinal; KPS, Karnofsky Performance Status; IPTW, inverse probability of treatment weighting.

\section{Supplementary Files}

This is a list of supplementary files associated with this preprint. Click to download.

- Suppfigure1.png

- Suppfigure2.png

- Suppfigure3.png 